\title{
Some Actuarial Formula of Life Insurance for Fuzzy Markets*
}

\author{
Qiaoyu Huang, Liang Lin, Tao Sun \\ College of Science, Guilin University of Technology, Guilin, China \\ E-mail:Lzcst135@163.com \\ Received May 13, 2011; revised June 16, 2011; accepted June 25, 2011
}

\begin{abstract}
This paper presents an actuarial model of life insurance for fuzzy markets based on Liu process. At first, some researches about an actuarial model of life insurance for stochastic market and concepts about fuzzy process have been reviewed. Then, an actuarial model of life insurance for fuzzy process is formulated.
\end{abstract}

Keywords: Fuzzy Actuarial Model, Fuzzy Process, Liu Process, Geometric Liu Process, Actuarial Formula

\section{Introduction}

The concept of fuzzy set was initiated by Zadeh [1] via membership function in 1965. In order to measure a fuzzy event, Liu and Liu [2] introduced the concept of credibility measure in 2002. Li and Liu [3] gave a sufficient and necessary condition for credibility measure in 2006 (cf. Liu [4]). Credibility theory was founded by Liu [5] in 2004 and refined by Liu [6] in 2007 as a branch of mathematics for studying the behavior of fuzzy phenomena. Credibility theory is deduced from the normality, monotonicity, self-duality, and maximality axioms. Liu [7] recently introduced the concepts of fuzzy process, Liu process and the geometric Liu process which will be commonly used model in finance for the value of an asset in a fuzzy environment. The two types of fundamental and important fuzzy processes, Liu process and the geometric Liu process, are the counterparts of Brownian motion and the geometric Brownian motion, respectively.

Li [8] presented an alternative assumption that stock price follows geometric Liu process. It is an application of fuzzy process for stock markets. Peng [9] presented A General Stock Model for Fuzzy Markets based on Liu's research in 2008. In 2010, Gao and Zhao [10] first presented fuzzy interests rate for insurance market.

The remainder of this paper is structured as follows. Section 2 is intended to introduce some useful concepts of fuzzy process as they are needed. Section 3 reviews Gao and Zhao's actuarial model for fuzzy market. An

*Supported by the Innovation Project of Guangxi Graduate Education (Grant No. 2011105960202M31). actuarial model of life insurance with payouts increased for fuzzy process is formulated in Section 4. Some actuarial formula of pure premium of the n-year continuous life insurance model is discussed in Section 5, as discount factor is a fuzzy process. Finally, some remarks are made in the concluding section.

\section{Preliminaries}

In this section, we will introduce some useful definitions and properties about fuzzy process.

\subsection{Fuzzy Process}

Definition 1. (Liu [7]) Given an index set $T$ and a credibility space $(\Theta, P, C r)$, a fuzzy process is a func-tion from $T \times(\Theta, P, C r)$ to the set of real numbers. In other words, a fuzzy process $X(t, \theta)$ is a function of two variables such that the function $X\left(t^{*}, \theta\right)$ is a fuzzy variable for each $t^{*}$. For simplicity, sometimes we simply use the symbol $X_{t}$ instead of longer notation $X(t, \theta)$.

Definition 2. (Liu [7]) A fuzzy process $X_{t}$ is said to have independent increments if

$$
X_{t_{1}}-X_{t_{0}}, X_{t_{2}}-X_{t_{1}}, \cdots, X_{t_{k}}-X_{t_{k-1}}
$$

are independent fuzzy variables for any times

$t_{0}<t_{1}<\cdots<t_{k}$. A fuzzy process $X_{t}$ is said to have stationary increments if, for any given $s>0$, the $X_{s+t}-X_{s}$ are identically distributed fuzzy variables for all $t>0$. 


\subsection{Liu Process}

Definition 3. (Liu [7]) A fuzzy process $C_{t}$ is said to be a Liu process if

1) $C_{o}=0$

2) $C_{t}$ has stationary and independent increments

3) every increment $C_{s+t}-C_{s}$ is a normally distributed fuzzy variable with expected value et and variance $\sigma^{2} t^{2}$, whose membership function is

$$
\mu(x)=2\left(1+\exp \left(\frac{\pi|x-u t|}{\sqrt{6} \sigma t}\right)\right)^{-1}, x \in R
$$

The parameters $e$ and $\sigma(>0)$ are called the drift and diffusion coefficients, respectively. The Liu process is said to be standard if $e=0$ and $\sigma=1$. The Liu process plays the role of the counterpart of Brownian motion.

Definition 4. (Liu [7]) Let $C_{t}$ be a standard Liu process. Then the fuzzy process

$$
G_{t}=\exp \left(e t+\sigma C_{t}\right)
$$

is called a geometric Liu process, or sometimes exponential Liu process. Li [8] has deduced that $G_{t}$ is of a lognormal membership function

$$
\mu(x)=2\left(1+\exp \left(\frac{\pi|\ln x-e t|}{\sqrt{6} \sigma t}\right)\right)^{-1}, x \geq 0
$$

and the expected value is

$E[\xi]=e^{e} \sqrt{6} \sigma \csc (\sqrt{6} \sigma), \sigma<\pi / \sqrt{6}$ had been proved by $\mathrm{Li}$ and Qin [8].

\section{Fuzzy Model of Discount Factor}

In this section we review a fuzzy model of discount factor which presented by Gao and Zhao, the model is assumed that interests rate is a trapezoidal fuzzy variables.

Accumulation function for force of interest is $y(t)$, as insurer, $V_{T}$ is present value of payment at the end of $m$ year. As insured, the present value for Survival annuity paid 1 per year is $Y_{m}$.

$$
\begin{aligned}
& y(t)=\delta t, 0 \leq t \leq n \\
& V_{T}= \begin{cases}e^{-y(K-1-m)}, & m \leq K<n \\
0 & , K \geq n\end{cases}
\end{aligned}
$$

where $\delta$ is a trapezoidal fuzzy variable $(a, b, c, d)$, $a \leq b \leq c \leq d$.

\section{An Actuarial Model for Fuzzy Process}

\subsection{The Model}

The paper hypothesizes that interest rate has two com- ponents: risk-free interest and risk interest. $\delta$ is used to represent force of interest, $y(t)=\delta_{0} t+\beta C_{t}$ is the accumulation function for force of interest, where $\delta_{0}$ is a part of interests with no risk, $C_{t}$ is a standard Liu process, used to describe the interests on risk. $\beta$ is $\beta$-coefficient.

We have the discount factor:

$$
V_{t}=\exp (-y(t))
$$

The actuarial model for Liu process is:

$$
\begin{aligned}
& y(t)=\delta_{0} t+\beta C_{t} \\
& V_{t}=\exp (-y(t))
\end{aligned}
$$

\subsection{Expected Value for $V_{t}$}

$C_{t}$ is a normally distribution fuzzy variable

$C_{t} \sim N\left(e t, \sigma^{2} t^{2}\right)$. Because $C_{t}$ is a standard Liu process with $e=0, \sigma=1, C_{t} \sim N\left(0, t^{2}\right)$. If $t \beta<\pi / \sqrt{6}$, then it is easy to prove that the expected value is

$$
\begin{aligned}
E\left(V_{t}\right)= & E\left[\exp \left(-\delta_{0} t-\beta C_{t}\right)\right] \\
& =e^{-\delta_{0} t} E\left(e^{-\beta C_{t}}\right) \\
& =e^{-\delta_{0} t}\left(\int_{0}^{+\infty} \operatorname{Cr}\left\{e^{-\beta \xi} \geq x\right\} \mathrm{d} x-\int_{-\infty}^{0} \operatorname{Cr}\left\{e^{-\beta \xi} \leq x\right\} \mathrm{d} x\right) \\
& =e^{-\delta_{0} t} \int_{0}^{\infty} \operatorname{Cr}\left\{\xi \leq \frac{1}{\beta} \ln x\right\} \mathrm{d} x \\
& =e^{-\delta_{0} t} \int_{0}^{\infty} 1-\left(1+\exp \left(-\frac{\pi\left(\frac{1}{\beta} \ln x\right)}{\sqrt{6} t}\right)\right)^{-1} \mathrm{~d} x \\
& =e^{-\delta_{0} t} \int_{0}^{\infty} 1-\frac{1}{1+x} \mathrm{~d} x \\
& =e^{-\delta_{0} t} \int_{0}^{\infty} \frac{1}{\sqrt{\sqrt{6} t \beta})} \mathrm{d} x \\
& =e^{-\delta_{0} t} \sqrt{6} \beta t \csc (\sqrt{6} \beta t)
\end{aligned}
$$

Otherwise, we have $E\left(V_{t}\right)=\infty$.

\section{Some Actuarial Formula of Pure Premium of the N-Year Continuous Life Insurance}

The pure premium of the n-year continuous life insur- 
ance with payment $b(t)$ is

$$
\begin{aligned}
\bar{A}_{x: \bar{n}}^{1} & =E\left[V_{T}\right]=\int_{0}^{n} b(t) E\left[V_{t}\right]_{t} p_{x} \mu_{x+t} \mathrm{~d} t \\
& =\int_{0}^{n} b(t) e^{-\delta_{0} t} \sqrt{6} \beta t \csc (\sqrt{6} \beta t){ }_{t} p_{x} \mu_{x+t} \mathrm{~d} t
\end{aligned}
$$

where $\beta t<\pi / \sqrt{6}$

\subsection{Pure Premium of the N-Year Continuous Life Insurance under the Assumption of de Moivre}

Under the assumption of de Moivre, we have

$$
\mu_{x+t}=\frac{1}{\omega-x-t},{ }_{t} p_{x} \mu_{x+t}=\frac{1}{\omega-x}
$$

Then

$$
\begin{aligned}
\bar{A}_{x: \bar{n}}^{1} & =\int_{0}^{n} b(t) e^{\delta_{0} t} \sqrt{6} \beta t \csc (\sqrt{6} \beta t){ }_{t} p_{x} \mu_{x+t} \mathrm{~d} t \\
& =\frac{\sqrt{6} \beta}{\omega-x} \int_{0}^{n} t b(t) e^{-\delta_{0} t} \csc (\sqrt{6} \beta t) \mathrm{d} t, \beta t<\pi / \sqrt{6}
\end{aligned}
$$

where $\bar{A}_{x: \bar{n}}^{1}$ respected to pure premium of the n-year continuous life insurance.

If the compensation is a constant, that is to say $b(t)=b$, so we have

$$
\begin{aligned}
\bar{A}_{x: \bar{n}}^{1} & =b \int_{0}^{n} e^{-\delta_{0} t} \sqrt{6} \beta t \csc (\sqrt{6} \beta t){ }_{t} p_{x} \mu_{x+t} \mathrm{~d} t \\
& =\frac{\sqrt{6} \beta b}{\omega-x} \int_{0}^{n} t e^{-\delta_{0} t} \csc (\sqrt{6} \beta t) \mathrm{d} t, \beta t<\pi / \sqrt{6}
\end{aligned}
$$

If the payment amount is increasing linearly, $b(t)=b+t$, then

$$
\begin{aligned}
& \begin{aligned}
I \bar{A}_{x: \bar{n}}^{1} & =\int_{0}^{n}(b+t) e^{-\delta_{0} t} \sqrt{6} \beta t \csc (\sqrt{6} \beta t){ }_{t} p_{x} \mu_{x+t} \mathrm{~d} t \\
& =\frac{\sqrt{6} \beta}{\omega-x} \int_{0}^{n}(b+t) t e^{-\delta_{0} t} \csc (\sqrt{6} \beta t) \mathrm{d} t
\end{aligned} \\
& \beta t<\pi / \sqrt{6}
\end{aligned}
$$

If the payment amount is increasing geometrically, $b(t)=t^{k}$,

$$
\begin{aligned}
& \begin{aligned}
\bar{A}_{x: \bar{n}}^{1} & =\int_{0}^{n} t^{k} e^{-\delta_{0} t} \sqrt{6} \beta t \csc (\sqrt{6} \beta t)_{t} p_{x} \mu_{x+t} \mathrm{~d} t \\
& =\frac{\sqrt{6} \beta}{\omega-x} \int_{0}^{n} t^{k+1} e^{-\delta_{0} t} \csc (\sqrt{6} \beta t) \mathrm{d} t
\end{aligned} \\
& \beta t<\pi / \sqrt{6}
\end{aligned}
$$

If the payment amount is increasing exponentially, $b(t)=e^{\alpha t}$,

$$
\begin{aligned}
\begin{aligned}
\bar{A}_{x: \bar{n}}^{1} & =\int_{0}^{n} e^{\alpha t} e^{-\delta_{0} t} \sqrt{6} \beta t \csc (\sqrt{6} \beta t){ }_{t} p_{x} \mu_{x+t} \mathrm{~d} t \\
& =\frac{\sqrt{6} \beta}{\omega-x} \int_{0}^{n} t e^{\left(\alpha-\delta_{0}\right) t} \csc (\sqrt{6} \beta t) \mathrm{d} t
\end{aligned} \\
\beta t<\pi / \sqrt{6}
\end{aligned}
$$

\subsection{Pure Premium of the N-Year Continuous Life Insurance under the Assumption of Gompertz}

Under the assumption of Gompertz, $\mu_{x+t}=B C^{x+t}$, $B>0, \quad C \geq 1$, and

$$
{ }_{t} p_{x} \mu_{x+t}=B C^{x+t} e^{\frac{B}{\ln C} C^{x}\left(1-C^{t}\right)}
$$

If the compensation is a constant, that is to say $b(t)=b$, so we have

$$
\begin{aligned}
\bar{A}_{x: \bar{n}}^{1}= & \int_{0}^{n} b(t) e^{-\delta_{0} t} \sqrt{6} \beta t \csc (\sqrt{6} \beta t)_{t} p_{x} \mu_{x+t} \mathrm{~d} t \\
= & \sqrt{6} \beta b \int_{0}^{n} t e^{-\delta_{0} t} \csc (\sqrt{6} \beta t) B C^{x+t} e^{\frac{B}{\ln C} C^{x}\left(1-C^{t}\right)} \mathrm{d} t \\
& =\sqrt{6} \beta b B \int_{0}^{n} t C^{x+t} e^{-\delta_{0} t+\frac{B}{\ln C} C^{x}\left(1-C^{t}\right)} \csc (\sqrt{6} \beta t) \mathrm{d} t \\
\beta t< & \pi / \sqrt{6}
\end{aligned}
$$

If the payment amount is increasing linearly, $b(t)=b+t$, then

$$
\begin{aligned}
& \begin{aligned}
& I \bar{A}_{x: \bar{n}}^{1}=\int_{0}^{n} b(t) e^{-\delta_{0} t} \sqrt{6} \beta t \csc (\sqrt{6} \beta t){ }_{t} p_{x} \mu_{x+t} \mathrm{~d} t \\
&=\sqrt{6} \beta \int_{0}^{n}(b+t) t e^{-\delta_{0} t} \csc (\sqrt{6} \beta t) B C^{x+t} e^{\frac{B}{\ln C} C^{x}\left(1-C^{t}\right)} \mathrm{d} t \\
&=\sqrt{6} \beta B \int_{0}^{n}(b+t) t C^{x+t} e^{-\delta_{0} t+\frac{B}{\ln C} C^{x}\left(1-C^{t}\right)} \csc (\sqrt{6} \beta t) \mathrm{d} t \\
& \beta t<\pi / \sqrt{6}
\end{aligned}
\end{aligned}
$$

If the payment amount is increasing geometrically, $b(t)=t^{k}$,

$$
\begin{aligned}
\bar{A}_{x: \bar{n}}^{1} & =\int_{0}^{n} b(t) e^{-\delta t} \sqrt{6} \beta t \csc (\sqrt{6} \beta t){ }_{t} p_{x} \mu_{x+t} \mathrm{~d} t \\
& =\sqrt{6} \beta \int_{0}^{n} t^{k} t e^{-\delta t} \csc (\sqrt{6} \beta t) B C^{x+t} e^{\frac{B}{\ln C} C^{x}\left(1-C^{t}\right)} \mathrm{d} t \\
& =\sqrt{6} \beta B \int_{0}^{n} t^{k+1} C^{x+t} e^{-\delta t+\frac{B}{\ln C} C^{x}\left(1-C^{t}\right)} \csc (\sqrt{6} \beta t) \mathrm{d} t \\
\beta t & <\pi / \sqrt{6}
\end{aligned}
$$

If the payment amount is increasing exponentially, $b(t)=e^{\alpha t}$,

$$
\begin{aligned}
\bar{A}_{x: \bar{n}}^{1} & =\int_{0}^{n} b(t) e^{-\delta_{0} t} \sqrt{6} \beta t \csc (\sqrt{6} \beta t){ }_{t} p_{x} \mu_{x+t} \mathrm{~d} t \\
& =\sqrt{6} \beta \int_{0}^{n} e^{\alpha t} t e^{-\delta_{0} t} \csc (\sqrt{6} \beta t) B C^{x+t} e^{\frac{B}{\ln C} C^{x}\left(1-C^{t}\right)} \mathrm{d} t \\
& =\sqrt{6} \beta B \int_{0}^{n} C^{x+t} e^{\left(\alpha-\delta_{0}\right) t+\frac{B}{\ln C} C^{x}\left(1-C^{t}\right)} \csc (\sqrt{6} \beta t) \mathrm{d} t \\
\beta t & <\pi / \sqrt{6}
\end{aligned}
$$




\subsection{Pure Premium of the $\mathbf{N}$-Year Continuous Life Insurance under the Assumption of Makeham}

Under the assumption of Makeham,

$$
\begin{gathered}
\mu_{x+t}=A+B C^{x+t}, B>0, C \geq 1, A \geq-B \\
{ }_{t} p_{x} \mu_{x+t}=\left(A+B C^{x+t}\right) e^{-A t+\frac{B}{\ln C} C^{x}\left(1-C^{t}\right)}
\end{gathered}
$$

If the compensation is a constant, that is to say $b(t)=b$, so we have

$$
\begin{aligned}
& \bar{A}_{x: \bar{n}}^{1}= \int_{0}^{n} b(t) e^{-\delta_{0} t} \sqrt{6} \beta t \csc (\sqrt{6} \beta t)_{t} p_{x} \mu_{x+t} \mathrm{~d} t \\
&= \sqrt{6} \beta b \int_{0}^{n} t e^{-\delta_{0} t} \csc (\sqrt{6} \beta t)\left(A+B C^{x+t}\right) \\
& \cdot e^{-A t+\frac{B}{\ln C} C^{x}\left(1-C^{t}\right)} \mathrm{d} t=\sqrt{6} \beta b \int_{0}^{n} t\left(A+B C^{x+t}\right) \\
& \cdot e^{-\left(\delta_{0}+A\right) t+\frac{B}{\ln C} C^{x}\left(1-C^{t}\right)} \csc (\sqrt{6} \beta t) \mathrm{d} t \\
& \beta t<\pi / \sqrt{6}
\end{aligned}
$$

If the payment amount is increasing linearly, $b(t)=b+t$, then

$$
\begin{aligned}
& \bar{A}_{x: \bar{n}}^{1}=\int_{0}^{n} b(t) e^{-\delta_{0} t} \sqrt{6} \beta t \csc (\sqrt{6} \beta t){ }_{t} p_{x} \mu_{x+t} \mathrm{~d} t \\
&=\sqrt{6} \beta \int_{0}^{n}(b+t) t e^{-\delta_{0} t} \csc (\sqrt{6} \beta t)\left(A+B C^{x+t}\right) \\
& \cdot e^{-A t+\frac{B}{\ln C} C^{x}\left(1-C^{t}\right)} \mathrm{d} t=\sqrt{6} \beta \int_{0}^{n} t(b+t)\left(A+B C^{x+t}\right) \\
& \cdot e^{-\left(\delta_{0}+A\right) t+\frac{B}{\ln C} C^{x}\left(1-C^{t}\right)} \csc (\sqrt{6} \beta t) \mathrm{d} t \\
& \beta t<\pi / \sqrt{6}
\end{aligned}
$$

If the payment amount is increasing geometrically, $b(t)=t^{k}$

$$
\begin{aligned}
\bar{A}_{x: \bar{n}}^{1} & =\int_{0}^{n} b(t) e^{\delta_{0} t} \sqrt{6} \beta t \csc (\sqrt{6} \beta t)_{t} p_{x} \mu_{x+t} \mathrm{~d} t \\
& =\sqrt{6} \beta \int_{0}^{n} t^{k} t e^{\delta_{0} t} \csc (\sqrt{6} \beta t)\left(A+B C^{x+t}\right) \\
& \cdot e^{-A t+\frac{B}{\ln C} C^{x}\left(1-C^{t}\right)} \mathrm{d} t=\sqrt{6} \beta \int_{0}^{n} t^{k+1}\left(A+B C^{x+t}\right) \\
& \cdot e^{-\left(\delta_{0}+A\right) t+\frac{B}{\ln C} C^{x}\left(1-C^{t}\right)} \csc (\sqrt{6} \beta t) \mathrm{d} t \\
\beta t< & \pi / \sqrt{6}
\end{aligned}
$$

If the payment amount is increasing exponentially, $b(t)=e^{\alpha t}$

$$
\begin{aligned}
\bar{A}_{x: \bar{n}}^{1} & =\int_{0}^{n} b(t) e^{\delta_{0} t} \sqrt{6} \beta t \csc (\sqrt{6} \beta t)_{t} p_{x} \mu_{x+t} \mathrm{~d} t \\
& =\sqrt{6} \beta \int_{0}^{n} e^{\alpha t} t e^{-\delta_{0} t} \csc (\sqrt{6} \beta t)\left(A+B C^{x+t}\right) \\
& \cdot e^{-A t+\frac{B}{\ln C} C^{x}\left(1-C^{t}\right)} \mathrm{d} t \sqrt{6} \beta \int_{0}^{n} t\left(A+B C^{x+t}\right) \\
& \cdot e^{\left(\alpha-\delta_{0}-A\right) t+\frac{B}{\ln C} C^{x}\left(1-C^{t}\right)} \csc (\sqrt{6} \beta t) \mathrm{d} t \\
\beta t< & \pi / \sqrt{6}
\end{aligned}
$$

\subsection{Pure Premium of the N-Year Continuous Life Insurance under the Assumption of Weibull}

Under the assumption of Weibull ,

$$
\begin{gathered}
\mu_{x+t}=k(x+t)^{n}, k>0, n>0 \\
{ }_{t} p_{x} \mu_{x+t}=k(x+t)^{n} e^{k(n+1)\left[x^{n+1}-(x+t)^{n+1}\right]}
\end{gathered}
$$

If the compensation is a constant, that is to say $b(t)=b$, so we have

$$
\begin{aligned}
\bar{A}_{x: \bar{n}}^{1}= & \int_{0}^{n} b(t) e^{-\delta_{0} t} \sqrt{6} \beta t \csc (\sqrt{6} \beta t)_{t} p_{x} \mu_{x+t} \mathrm{~d} t \\
= & \sqrt{6} \beta b \int_{0}^{n} t e^{-\delta_{0} t} \csc (\sqrt{6} \beta t) k(x+t)^{n} \\
& \cdot e^{k(n+1)\left[x^{n+1}-(x+t)^{n+1}\right]} \mathrm{d} t=\sqrt{6} \beta b k e^{k(n+1) x^{n+1}} \\
& \cdot \int_{0}^{n} t(x+t)^{n} e^{-\delta_{0} t-k(n+1)(x+t)^{n+1}} \csc (\sqrt{6} \beta t) \mathrm{d} t \\
\beta t< & \pi / \sqrt{6}
\end{aligned}
$$

If the payment amount is increasing linearly, $b(t)=b+t$, then

$$
\begin{aligned}
\bar{A}_{x: \bar{n}}^{1}= & \int_{0}^{n} b(t) e^{-\delta_{0} t} \sqrt{6} \beta t \csc (\sqrt{6} \beta t)_{t} p_{x} \mu_{x+t} \mathrm{~d} t \\
= & \sqrt{6} \beta \int_{0}^{n} t(b+t) e^{-\delta_{0} t} \csc (\sqrt{6} \beta t) k(x+t)^{n} \\
& \cdot e^{k(n+1)\left[x^{n+1}-(x+t)^{n+1}\right]} \mathrm{d} t=\sqrt{6} \beta k e^{k(n+1) x^{n+1}} \\
& \cdot \int_{0}^{n} t(b+t)(x+t)^{n} e^{-\delta_{0} t-k(n+1)(x+t)^{n+1}} \csc (\sqrt{6} \beta t) \mathrm{d} t \\
\beta t< & \pi / \sqrt{6}
\end{aligned}
$$

If the payment amount is increasing geometrically, $b(t)=t^{a}$

$$
\begin{aligned}
\bar{A}_{x: \bar{n}}^{1}= & \int_{0}^{n} b(t) e^{-\delta_{0} t} \sqrt{6} \beta t \csc (\sqrt{6} \beta t)_{t} p_{x} \mu_{x+t} \mathrm{~d} t \\
= & \sqrt{6} \beta \int_{0}^{n} t t^{a} e^{-\delta_{0} t} \csc (\sqrt{6} \beta t) k(x+t)^{n} \\
& \cdot e^{k(n+1)\left[x^{n+1}-(x+t)^{n+1}\right]} \mathrm{d} t=\sqrt{6} \beta k e^{k(n+1) x^{n+1}}
\end{aligned}
$$$$
\cdot \int_{0}^{n} t^{a+1}(x+t)^{n} e^{-\delta_{0} t-k(n+1)(x+t)^{n+1}} \csc (\sqrt{6} \beta t) \mathrm{d} t
$$

$\beta t<\pi / \sqrt{6}$ 
If the payment amount is increasing exponentially, $b(t)=e^{\alpha t}$

$$
\begin{aligned}
\bar{A}_{x: \bar{n}}^{1}= & \int_{0}^{n} b(t) e^{-\delta_{0} t} \sqrt{6} \beta t \csc (\sqrt{6} \beta t)_{t} p_{x} \mu_{x+t} \mathrm{~d} t \\
= & \sqrt{6} \beta \int_{0}^{n} t e^{\alpha t} e^{-\delta_{0} t} \csc (\sqrt{6} \beta t) k(x+t)^{n} \\
& \cdot e^{k(n+1)\left[x^{n+1}-(x+t)^{n+1}\right]} \mathrm{d} t=\sqrt{6} \beta k e^{k(n+1) x^{n+1}} \\
& \cdot \int_{0}^{n} t(x+t)^{n} e^{\left(\alpha-\delta_{0}\right) t-k(n+1)(x+t)^{n+1}} \csc (\sqrt{6} \beta t) \mathrm{d} t \\
\beta t< & \pi / \sqrt{6}
\end{aligned}
$$

\section{Conclusions}

The main contribution of this paper is to suggest a new actuarial for fuzzy markets by means of Liu process. Some actuarial formulas of pure premium of the n-year continuous life insurance on the proposed fuzzy model are investigated.

\section{References}

[1] L. A. Zadeh, "Fuzzy Sets," Information and Control, Vol. 8, No. 3, 1965, pp. 338-353. doi:10.1016/S0019-9958(65)90241-X

[2] B. D. Liu and Y. K. Liu, "Expected Value of Fuzzy Vari- able and Fuzzy Expected Value Models,” IEEE Transactions on Fuzzy Systems, Vol. 10, No. 4, 2002, pp. 445-450. doi:10.1109/TFUZZ.2002.800692

[3] X. Li and B. D. Liu, "A Sufficient and Necessary Condition for Credibility Measures," International Journal of Uncertainty, Fuzziness \& Knowledge-Based Systems, Vol. 14, No. 5, 2006, pp. 527-535. doi:10.1142/S0218488506004175

[4] B. D. Liu, “Uncertainty Theory,” Springer-Verlag, Berlin, 2004.

[5] B. D. Liu, "Uncertainty Theory," 2nd Edition, SpringerVerlag, Berlin, 2007.

[6] B. D. Liu, “A Survey of Credibility Theory,” Fuzzy Optimization and Decision Making, Vol. 5, No. 4, 2006, pp. 387-408. doi:10.1007/s10700-006-0016-x

[7] B. D. Liu, "Fuzzy Process, Hybrid Process and Uncertain Process," Journal of Uncertain Systems, Vol. 2, No. 1, 2008, pp. 3-16.

[8] X. Li and F. Q. Zhong, "Eepected Value and Variance of Geometric Liu Process,” Far East Journal of Experimental and Theoretical Artificial Intelligence, Vol. 1, No. 2, 2008, pp. 127-135.

[9] J. Peng, “A General Stock Model for Fuzzy Markets,” Journal of Uncertain Systems, Vol. 2, No. 4, 2008, pp. 248-254.

[10] J. G. Gao and M. Q. Zhao, "The Life Insurance Model for Fuzzy Interest Rate (Chinese),” Journal of Systems Engineering, Vol. 25, 2010, pp. 603-608. 